\title{
Deshidratacion de los alcoholes presentes en el aceite fusel y su conversión en alquenos empleando una zeolita tipo HZSM-5
}

CHEMICAL ENGINEERING

\section{Dehydration of alcohols present in fusel oil and its conversion to alkenes using a HZSM-5 zeolite}

\author{
Julián D. Urresta§ \\ Laboratorio de Investigación en Catálisis y Procesos LICAP. Departamento de Química. Facultad de \\ Ingenieria, Universidad del Valle, Cali, Colombia \\ §julian.urresta@correounivalle.edu.co
}

(Recibido: 24 de enero de 2013 -Aceptado: 23 de mayo de 2014)

\begin{abstract}
Resumen
El aceite fusel es un sub-producto de las destilerías durante la preparación del etanol mediante la fermentación del azúcar. En este trabajo se muestran los resultados de la transformación catalítica en alquenos de los alcoholes presentes en el aceite fusel, empleando dos zeolitas pertenecientes a la familia de los pentasil como la MFI y la HZSM-5 con relación Si/Al 28 y 1000, buscando obtener alquenos como: el buteno o el isopenteno, empleados ampliamente en la industria como bio-lubricante. Se encontró que el sistema más activo hacia la obtención de alquenos fue la zeolita HZSM-5 relación $\mathrm{SiO}_{2} / \mathrm{Al}_{2} \mathrm{O}_{3}=28$. El análisis de los productos de reacción se realizó por cromatografía gaseosa con detector FID encontrándose que a $200^{\circ} \mathrm{C}$ se obtiene una transformación del $90 \%$ y solo al elevar la temperatura hasta $\operatorname{los} 260^{\circ} \mathrm{C}$ se logra la conversión total. Por otro lado, todos los catalizadores presentaron una desactivación rápida y la actividad cayó drásticamente al cabo de 24 horas.
\end{abstract}

Palabras claves: Aceite fusel, alquenos, deshidratación, zeolita ácida

\begin{abstract}
Fusel oil is a by-product of distilleries during the preparation of ethanol by fermentation of sugar. In this paper is shown the results of the catalytic transformation in alkenes of the alcohols present in fusel oil, using two zeolites belonging to the family of Pentasil as MFI and HZSM -5 with Si / Al 28 and 1000, looking for obtaining alkenes such as butene or isopentene widely employed in industry as a bio-lubricant. We found that the most active system towards obtaining alkenes was the HZSM-5 zeolite ratio $\mathrm{SiO}_{2} / \mathrm{Al}_{2} \mathrm{O}_{3}=28$. The analysis of the reaction products was carried out by gas chromatography with FID detector was found that at $200{ }^{\circ} \mathrm{C} 90 \%$ transformation is obtained and only to raise the temperature to $260{ }^{\circ} \mathrm{C}$ complete conversion is achieved. Furthermore, all of the catalysts showed a rapid deactivation and the activity dropped sharply after 24 hours.
\end{abstract}

Keywords: Acidic zeolite, alkenes, dehydration, fusel oil. 


\section{Introducción}

$\mathrm{El}$ aceite de fusel corresponde al residuo que queda después de destilación de alcohol fermentado y se compone de una mezcla de alcoholes, de $\mathrm{C}_{2}$ a $\mathrm{C}_{5}$, lo que representa una fuente barata y renovable de mezcla de alcoholes ligeros (alcohol isoamílico principalmente), obtenidos a partir de la producción de etanol, la cual es una industria en rápido crecimiento Cruz (2013). El aceite de fusel se puede derivar a partir de bioetanol de primera generación (azúcar o almidón) y también de segunda generación (biomasa lignocelulósica). Hasta ahora, sólo una pequeña cantidad de aceite de fusel se recupera con fines comerciales (aroma en la industria alimentaria y de la perfumería). Los alcoholes presentes en aceites de fusel son entre el 50 y $70 \%$ de la mezcla, en función del origen, ver tabla 1.

En cuanto al alcohol isoamílico, principal componente del aceite fusel, es un compuesto orgánico que tiene una fórmula molecular $\mathrm{C}_{5} \mathrm{H}_{12} \mathrm{O}$. Es un líquido incoloro, de volatilidad moderada y es aproximadamente cuatro veces más tóxico que el etanol. Se usa como disolvente en tintas de impresión, lacas, gomas o para la fabricación de intermediarios químicos en reacciones de esterificación. El mayor uso del alcohol isoamílico es en la producción del acetato de amilo (esencia de banano), para la industria de las lacas, perfumería y fragancias.

La transformación del aceite de fusel en productos de mayor valor agregado ha sido estudiada desde hace tiempo. Es así como la vía biotecnológica para obtener bio-productos (entre ellos los biocombustibles) ha sido estudiada por varios investigadores Sun et al. (2012), Watanabe et al. (2000), De Castro et al. (1999b), Dôrmô et al. (2004). En ellos se muestra la síntesis de un biolubricante preparado en el sistema integrado por una reacción de esterificación del aceite de fusel y el ácido oleico, utilizando para ello una enzima lipasa como biocatalizador y sin el empleo de solvente. En esa misma dirección, otras investigaciones se han efectuado para utilizarlo como aditivo mejorando el octanaje de la gasolina o para la producción de aromas naturales y lubricantes Welsh et al. (1989), Özgülsün et al. (1999a) y (2000b). Recientemente se le ha dado importancia al estudio de obtención de los biosolventes (esteres a base de productos provenientes de fuentes de origen vegetal o animales), los cuales ofrecen amplias ventajas para remplazar a los derivados de recursos fósiles. Las principales clases de biosolventes son esteres de ácidos orgánicos naturales (producido por la fermentación de materiales renovables), esteres de ácidos grasos, el bioetanol y el glicerol y sus derivados Bewa \& Alfos (2005), así como empleando enzimas, principalmente lipasas Welsh et al. (1989), De Castro et al. (1995a), Güvenc et al. (2007) y Vulfson (1993).

Los procesos industriales en reacciones de esterificación usualmente involucran catalizadores heterogéneos (ácidos fuertes como el ácido sulfúrico) entre los que se encuentran las resinas (e.g. Amberlyst-15) y los superácidos

Tabla 1 Composición (porcentaje másico de alcoholes) de varios aceites fusel.

\begin{tabular}{ccccc}
\hline $\begin{array}{c}\text { Origen \& Proveedor } \\
\text { de aceite Fusel }\end{array}$ & Beet Tereos & Beet UNGDAa & Wheat UNGDA & Crystal Union \\
\hline Etanol (\%) & 1.69 & 11.32 & 17.65 & 11 \\
Propanol (\%) & 0.03 & 0.08 & 0.09 & 0.5 \\
Isopropanol (\%) & $<0.01$ & 0.05 & $<0.01$ & $<0.1$ \\
Butanol (\%) & 0.14 & 0.06 & 0.15 & 4.0 \\
Isobutanol (\%) & 3.43 & 0.25 & 0.69 & 0.4 \\
C $_{5}$ alcohol (\%)b & 74.84 & 75.73 & 57.62 & 75 \\
Total (\%)c & 80.13 & 87.49 & 76.20 & 86.2 \\
\hline
\end{tabular}

a UNGDA: Unión nacional de destiladores de alcoholes (Francia).

$b$ Alcoholes $C_{5}$ corresponde a la mezcla de alcohol isoamílico (3-metil-1-butanol) and alcohol amílico (2-metil-1-butanol).

c Contenido de agua hasta alcanzar el 100\%. 
como zirconia sulfatada, así como la zeolita BEA (beta, H-BEA), la cual se ha empleado en la esterificación del acido acético con butanol, Peters et al. (2006) y Jermy et al. (2005). Hoek et al. (2004) aplicaron con éxito la zeolita BEA como catalizador para la reacción de eterificación del 1-octanol, mientras que Beers et al. (2001), y Nijhuis et al. (2002) investigaron el empleo de la zeolita BEA en la esterificación del ácido hexanoico con 1-octanol.

En particular, en catálisis heterogénea, muchos métodos sintéticos han sido desarrollados para la preparación delacetato de isoamilo. Tales métodos se han centrado en el empleo de condiciones suaves y a temperatura ambiente Dalpozzo et al. (2003), empleando resinas catiónicas Teo \& Saha (2004) y de otros catalizadores Pizzio \& Blanco (2003). Es necesario también, destacar el trabajo desarrollado por Bandres et al. (2011) en el LCA de Toulouse, en donde usan el alcohol isoamílico obtenido del aceite fusel para la producción de esteres, empleando una resina de intercambio catiónica como la Amberlist 15. Este estudio ha demostrado que un co-producto de la industria del bioetanol se puede utilizar como molécula de partida para producir compuestos más seguros en la sustitución de disolventes que se utilizan actualmente en la industria. Adicionalmente, se han efectuado estudios por Osorio-Viana et al. (2013) sobre el modelo cinético para la esterificación en fase líquida de ácido acético con alcohol isoamílico en presencia del catalizador heterogéneo Amberlite IR-120. En términos estadísticos, dos modelos cinéticos aparecen como los más apropiados para esta reacción de esterificación: un modelo simple fácil de manejar, que utiliza fracciones molares y un segundo modelo basado en el modelo de actividad NRTL, que es físicamente más realista debido a su congruencia con los fenómenos de sorción de resina. Es bien sabido que uno de los problemas para el uso industrial del aceite fusel es su purificación-separación y en ese sentido se espera que la destilación reactiva (RD), que es una estrategia de intensificación probada para la esterificación de alcoholes individuales también puede ser una opción prometedora para la esterificación simultánea de todos los alcoholes de aceite de fusel. Para evaluar su viabilidad y diseñar una unidad de gran escala, los modelos de equilibrio cinético y fase son necesarios y en ese sentido Patidar y Mahajani (2012), investigaron la cinética experimental de la reacción de reacciones de esterificación y transesterificación de los diferentes componentes del aceite de fusel con ácido acético, encontrando que un proceso viable basado en la destilación reactiva simulado en ASPEN PLUS usando la cinética desarrollada en dicho trabajo.

Otra vía interesante en la valorización del aceite fusel es la deshidratación catalítica de los alcoholes para la obtención de olefinas y aunque su interés había disminuido considerablemente desde que los hidrocarburos $\mathrm{C}_{2}$ se obtuvieron a partir de petróleo bruto, esta deshidratación es, sin embargo, un buen modelo para el estudio del mecanismo de eliminación empleando sistemas heterogéneos y para medir la actividad de los catalizadores, los cuales tienen propiedades esencialmente ácidas.

Las perspectivas de desarrollo de la química prevén el continuo incremento de la demanda de olefinas ligeras a corto término, las cuales se obtienen por rompimiento con vapor de la nafta o del gasoil. Además, la síntesis de productos oxigenados, principalmente a partir de alcoholes ligeros y polioles, puede resultar interesante, debido a las numerosas aplicaciones, por ejemplo, como sustituto de hidrocarburos, como carburante o como producto de base. Adicionalmente, en estos últimos años, la preocupación en el mundo para reducir la contaminación ambiental y sobre todo el aumento de la posición de la química verde muestran la vía del desarrollo de procesos más limpios y en particular para la utilización de materias primas obtenidas de fuentes renovables en lugar del petróleo.

Es necesario mencionar que en la década de los 70 se desarrollaron varios trabajos sobre la deshidratación de varios alcoholes como el tert-butanol catalizado por resinas, Frilette et al. (1964), Gates et al. (1972), así como la utilización de etanol y de n-butanol con zeolitas tipo Linde-X, Linde-A, \& Norton 
Zeolon, Bryant \& Kranich (1967), y mordenita Ignace \&Gates (1973). Por su parte Haering \& Syverson (1974), efectuaron un estudio sobre la adsorción del tert-butanol sobre una alúmina a $195^{\circ} \mathrm{C}$ a presión atmosférica. Los investigadores concluyen que el alcohol tert-butílico exhibe una rápida adsorción sobre la alúmina y la cantidad adsorbida del alcohol depende de la cantidad de agua adsorbida. Además, esta agua posee dos tipos de adsorción, una rápida reversible y una lenta, la cual se completa en 20 minutos y solo una parte es reversible. Por otro lado Bakshi \& Gavalas (1975) determinaron los calores de adsorción por titulación para alúminas comerciales, silica-aluminas y arcillas y así mismo relacionan la actividad catalítica en la deshidratación de alcoholes con la superficie ácida y básica del sólido.

En general, los estudios detallados en la literatura en la deshidratación de alcoholes se presentan sobre la $\delta$-alúmina Tanabe et al. (1989), Knözinger et al. (1972a), Shi et al. (1999), Gates (1992) y Makgoba et al. (2006) quienes han demostrado que las moléculas de alcohol están unidas a la superficie de alúmina a través de enlaces de hidrógeno, donde el alcohol y la superficie de alúmina tienen grupos funcionales complementarios, siendo a la vez un donante y un aceptor de protones. En este mecanismo propuesto, un protón se transfiere de un grupo hidroxilo a la superficie oxígeno alcohol, facilitando la transferencia de un protón en el carbono vecino a un átomo de oxígeno básico en la superficie. Más recientemente, otros sistemas catalíticos han sido estudiados como los óxidos a base de tungsteno para la deshidratación del 2-butanol por Baertsch et al. (2002). Ellos midieron los sitios Brønsted en catalizadores tipo $\mathrm{WO}_{\mathrm{x}}-\mathrm{ZrO}_{2}$, encontrando que la velocidad de deshidratación de 2-butanol (por átomo de $\mathrm{W}$ ) sobre $\mathrm{WO}_{\mathrm{x}}-\mathrm{ZrO}_{2}$ alcanza un valor máximo a densidades superficiales intermedias de WOx.

En este sentido, la investigación en síntesis orgánica de base y de hidrocarburos a partir de moléculas obtenidas de subproductos agrícolas ha sido relanzada y en los países donde el etanol carburante se produce a gran escala (en Colombia se produjeron en el 2012 cerca de 362 millones de litros / alcohol) Garcia (2013), la utilización de subproductos convierte la producción de etanol en menos contaminante y más rentable y en este trabajo se muestra que es posible lograr la transformación del aceite fusel en alquenos sin necesariamente efectuar una eliminación del agua presente en este compuesto.

\section{Procedimiento experimental}

\section{1 Materiales y catalizador para la síntesis}

El aceite fusel empleado fue donado por la empresa Crystal Union (Francia). El catalizador empleado en este estudio, una zeolita ZSM-5, la CBV 3204E con una relación $\mathrm{SiO}_{2} / \mathrm{Al}_{2} \mathrm{O}_{3}$ de 28 provista por Zeolyst. La zeolita fue secada en un horno a $353 \mathrm{~K}$ por $6 \mathrm{~h}$ y almacenada en un desecador hasta su uso. El catalizador posee un área específica de 42 $\mathrm{m}^{2} / \mathrm{g}$, determinada por el método de BET, con una capacidad de intercambio cercana a 4.8 meq $\mathrm{H}+/ \mathrm{g}$.

\subsection{Método de síntesis. Deshidratación heterogénea de aceite fusel}

El sistema está compuesto de un horno eléctrico en posición horizontal en el cual se introduce el reactor. El horno tiene un diámetro de $40 \mathrm{~mm}$ y la temperatura se regula gracias a un controlador acoplado a dos termocuplas térmicas tipo $\mathrm{K}$ situadas a nivel del lecho catalítico. El catalizador en polvo (HZSM-5) con relación $\mathrm{SiO}_{2} / \mathrm{Al}$ igual a 28, le confiere una elevada acidez. El catalizador está soportado por un tapón en lana de silicio y colocado en el medio del reactor y enseguida se fija el catalizador colocando lana de silicio encima.

Un perfusor Fischer Scientific modelo 78X1001, permite la introducción de la mezcla de reacción en el reactor, después de pasar en el tubo de precalentamiento para vaporizar la mezcla de alcoholes (aceite fusel). Las condiciones de inyección escogidas tratan de permitir la introducción de un pequeño flujo al interior del reactor (según tabla de diámetro de jeringas): número 7 Scientific glass engineering SGE $25 \mu \mathrm{L}$ et $0.73 \mathrm{~mm}$. Para inyectar los reactivos se utiliza una jeringa de $50 \mathrm{~mL}$ garantizando que el flujo 
de la mezcla de alcoholes sea lo suficientemente bajo para que los reactivos sean transformados y el escogido es de $0.5 \mathrm{~mL} / \mathrm{h}$. La introducción de un gas inerte $\left(\mathrm{N}_{2}\right)$ al inicio de la reacción se hace necesaria para llevar la mezcla a condiciones óptimas, siendo la relación $\mathrm{N}_{2} /$ aceite fusel de $18.7 / 1$.

Las condiciones de temperatura de reacción deben ser tales que la mayor parte de la mezcla de alcoholes se gasifique a la entrada del reactor. La temperatura de reacción debe permanecer entre 200 y $260^{\circ} \mathrm{C}$, por una parte para obtener una actividad importante y de otra parte para evitar ingresar una cantidad importante de líquido en el reactor. A la salida del reactor, los efluentes gaseosos son mantenidos a una temperatura comprendida entre 140 et $180^{\circ} \mathrm{C}$ con ayuda de un cordón calentador antes de entrar en la válvula de inyección en el cromatógrafo y de esta forma evitar la condensación de los efluentes. También se ha instalado una trampa para tratar de recuperar los productos condensables.

\subsection{Análisis cromatográfico}

Los resultados de reacción se determinan por medio de un cromatógrafo marca Perkin-Elmer con detector a llama de ionización (FID). La temperatura del inyector fue de $240^{\circ} \mathrm{C}$ y la del detector fue $250{ }^{\circ} \mathrm{C}$ con un rango de 1 y una atenuación de -3. La columna es una PORAPLOT $\mathrm{Q}$ de $10 \mathrm{~m}$ et $0.53 \mathrm{~mm}$ de diámetro. La rampa de temperatura es de $70^{\circ} \mathrm{C}$ durante $0.01 \mathrm{~min}$ para subir hasta $200^{\circ} \mathrm{C}$ a una velocidad de $10^{\circ} / \mathrm{min}$ y permanece por $10 \mathrm{~min}$. El gas de arrastre empleado es helio a una presión de 50 psi. El análisis de los efluentes se efectúa en línea con el cromatógrafo y la cantidad inyectada hacia el cromatógrafo se realiza por intermedio de una válvula de 8 vías. La identificación y la cuantificación de los productos gaseosos se efectúa con ayuda de un patrón (mezcla de metano, etano, etileno, $\mathrm{CO}$ y $\mathrm{CO}_{2}$ ) provisto por la Société Air Liquide.

Los tiempos de retención de los alcoholes fueron para el etanol 5.4 (minutos), isopropanol 6.8 (minutos), 1-Butanol 9.9 (minutos) y 1-Pentanol 13.9 (minutos).

\section{Resultados y discusión}

\subsection{Determinación de la composición y características del aceite de fusel}

En la tabla 1 se muestra los porcentajes de la composición para diferentes muestras del aceite de fusel comercial. El estado físico nos muestra que el aceite es un líquido de coloración amarilla clara y es soluble con un olor característico a productos de destilería. El valor del índice de acidez (0.808) indica que el aceite tiene poca cantidad de ácidos libres, como ácido acético.

\subsection{Estudio de la deshidratación de los alcoholes con la zeolita HZSM-5 relación $\mathrm{Si} / \mathrm{Al}=\mathbf{2 8}$}

En primer lugar se determinan los productos de deshidratación para los alcoholes puros (etanol, butanol y pentanol), los cuales están presentes en la mezcla de aceite fusel y de esta manera se puede tener una idea de los productos que se esperan encontrar en este estudio, así como de ver el comportamiento de la reacción, el flujo del gas vector y de los alcoholes, la temperatura necesaria para la deshidratación y sus tiempos de retención.

\subsubsection{Deshidratación del etanol y el butanol con la zeolita HZSM-5 relación $\mathrm{Si} / \mathrm{Al}=\mathbf{2 8}$}

Se encontró que para esta primera etapa, se debía de emplear una masa de catalizador del orden de $0.5 \mathrm{~g}$ en polvo, un flujo de alcohol de $0.3 \mathrm{~mL} / \mathrm{hora}$, con un flujo de gas de arrastre (nitrógeno) de $47 \mathrm{~mL} /$ minuto y la temperatura de ensayo de entre $140 \mathrm{y}$ $300^{\circ} \mathrm{C}$. Para la deshidratación del etanol, la cual fue efectuada a una temperatura de $200^{\circ} \mathrm{C}$, se encontró la presencia de un pico mayoritario a 2.6 minutos (etileno) y de un pico pequeño a 5.7 minutos (etanol). Algunos ensayos de larga duración se efectuaron sobre el catalizador HZSM-5 con y sin calcinación a $450^{\circ} \mathrm{C}$ y no se observó una variación de los resultados por este hecho.

Para el butanol, a $140^{\circ} \mathrm{C}$, se encontró la presencia de 3 picos representativos de la fracción hidrocarburos $\mathrm{C} 4$ a 5 minutos. Se constata igualmente la presencia de un pico a $9.7 \mathrm{~min}$ que pertenece al butanol. Cuando se trabajó a $260^{\circ} \mathrm{C}$, 
se observó la aparición de dos series de picos, dos a un tiempo de 3 minutos y 4 a 5 minutos. La serie a 3 minutos corresponde al propano - propeno, la serie a 5 minutos corresponde a los productos derivados del butano (1-buteno, isobuteno, cis 2-buteno, trans 2-buteno), los cuales se recopilan en la figura 1. Como se observa en la figura, el producto mayoritario el 1-buteno, mostrando estos resultados que la deshidratación de los alcoholes en presencia de catalizadores altamente ácidos, conlleva a la obtención de alquenos, en particular del 1-buteno.

La reacción de deshidratación de alcoholes es una reacción estereoeselectiva y en este caso la estereoselectividad de la reacción viene dada fundamentalmente por la estabilidad de los productos finales que se parecen en gran medida a la estabilidad de los intermedios carbocatiónicos y por tanto hay una tendencia a que se forme el producto más estable. Estos hechos sugieren que los carbocationes son los intermedios claves en el mecanismo de esta reacción. En la deshidratación de alcoholes primarios como es este caso, se formarían carbocationes primarios especies muy inestables para ser considerados como intermedios. Para los alcoholes primarios el protón se pierde del ión alquiloxonio en la misma etapa en la que tiene lugar la ruptura del enlace carbono-oxígeno estimándose que en este caso el mecanismo es E2 aunque transcurre muy lentamente. Estando lo anterior en concordancia con los resultados obtenidos por Berteau et al. (1991), puesto que el catalizador utilizado en este estudio corresponde a un sólido fuertemente ácido como la zeolita HZSM-5 con una relación silicio / aluminio de 28.

\subsubsection{Deshidración del pentanol con la zeolita HZSM-5 relación $\mathrm{Si} / \mathrm{Al}=\mathbf{2 8}$}

En la figura 2 se muestran los resultados de la deshidratación del pentanol cuando se trabajó a una temperatura de $200^{\circ} \mathrm{C}$. En ella se observa la transformación del alcohol en las series de hidrocarburos $\mathrm{C}_{2}, \mathrm{C}_{3}, \mathrm{C}_{4} \mathrm{y} \mathrm{C}_{5}$, siendo los productos de la serie $\mathrm{C}_{5}$ los que se producen en mayor cantidad con cerca del $86 \%$ en selectividad, sin que el catalizador se desactive durante la prueba de 20 horas. Se observa también la presencia de hidrocarburos asociados a la serie $\mathrm{C}_{5}$ (6 picos) para todas las inyecciones de la prueba. El producto principal es el que sale a $6.96 \mathrm{~min}$, que corresponde a la cis-2-penteno. De esta forma se lograron identificar los pentenos obtenidos, siendo el 3-metil-1-buteno quien sale primero a 5.86, seguido del 2-metil-1-buteno a 6.26, el 1-penteno a 6.49, el 2-metil-2-buteno a 6.74, el trans-2-penteno a 6.91 y el cis-2-penteno a 6.96 .

Teniendo en cuenta los resultados obtenidos y en paralelocon el trabajo efectuado porNorris \& Reuter

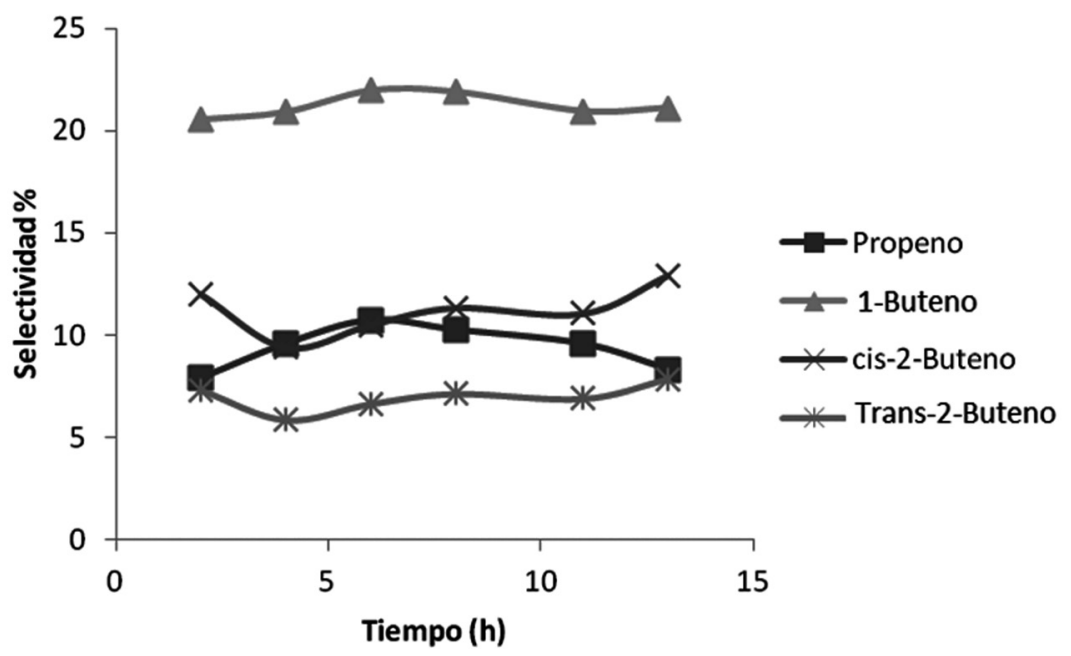

Figura 1. Evolución en función del tiempo de la deshidratación del butanol en presencia de la zeolita HZSM-5. Masa del catalizador $0.4995 \mathrm{~g}$ con $3.1221 \mathrm{~g}$ de carborundo y un flujo de inyección del alcohol de $0.5 \mathrm{~mL} / \mathrm{h}$ y un flujo de gas de arrastre de $\mathrm{N}_{2}$ de $28 \mathrm{~mL} / \mathrm{min}$. 


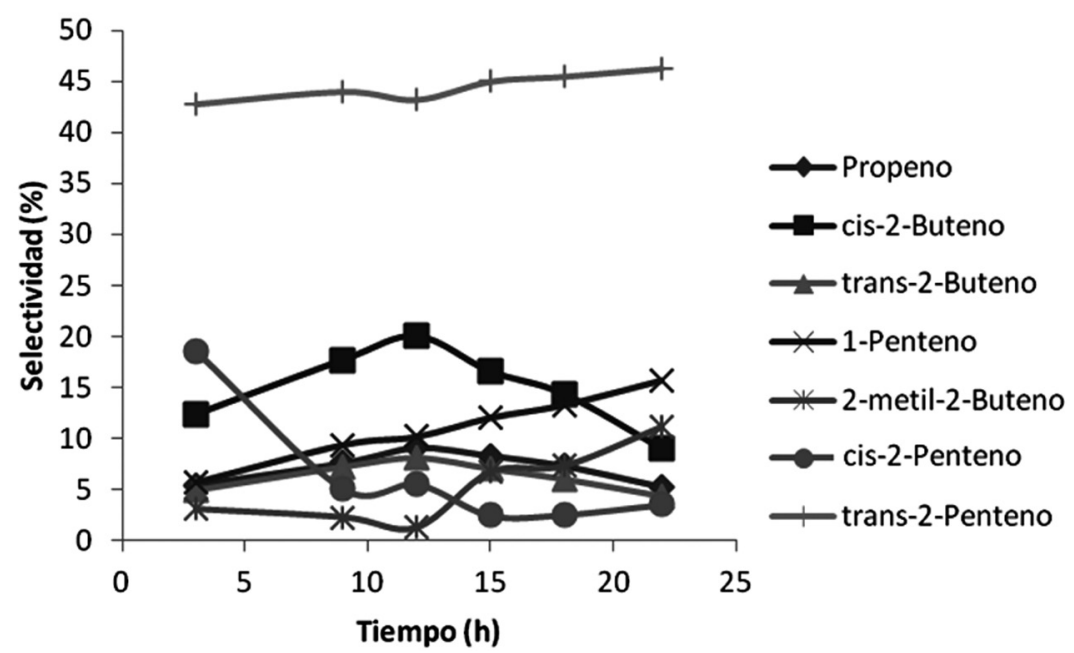

Figura 2. Evolución de la selectividad en función del tiempo para la deshidratación del pentanol. Masa del catalizador $0.4991 \mathrm{~g}$ con $3.0201 \mathrm{~g}$ de carborundo y un flujo de inyección del alcohol de $0.5 \mathrm{~mL} / \mathrm{h}$ y un flujo de gas de arrastre de $N_{2}$ de $28 \mathrm{~mL} / \mathrm{min}$.

(1927), los cuales reportaron un rendimiento del 90\% hacia el 3 metil-1-buteno, empleando alúmina como catalizador, es claro que existe un equilibrio entre los tres isómeros y según lo determinado por Ewell \& Hardy (1941) quienes dan cuenta que los calores de hidrogenación determinados a $80^{\circ} \mathrm{C}$ y expresados en kilocalorías/mole son: el 3 metil1-buteno $\mathrm{Q}=30,336$, el 2-metil-1-buteno $\mathrm{Q}=$ 28,491 y el 2-metil-2-buteno $\mathrm{Q}=26,920$, con lo cual se puede determinar que la estabilidad de los alquenos aumenta del 3 metil-1-buteno, al 2-metil1-buteno y al 2-metil-2-buteno. Para el caso del alcohol isoamílico presente mayoritariamente en el aceite fusel, su transformación conlleva a obtener mayoritariamente el trans-2-penteno, el cual desde el punto de vista termodinámico es más estable que el cis-2-penteno.

\subsection{Estudio de la deshidratación del aceite fusel con otros catalizadores}

\subsubsection{Zeolita HZSM-5 relación $\mathrm{Si} / \mathrm{Al}=\mathbf{1 0 0 0}$}

Cuando se utiliza una zeolita menos ácida con una relación $\mathrm{SiO}_{2} / \mathrm{Al}_{2} \mathrm{O}_{3}$ mayor (1000) la reacción de deshidratación del alcohol se inhibe, $\mathrm{y}$ es necesario aumentar la temperatura para una conversión de alcoholes en alquenos y en pequeñas cantidades. Para conseguir una buena transformación es necesario elevar la temperatura al menos hasta los $400^{\circ} \mathrm{C}$.

\subsubsection{Zeolita MFI}

Este catalizador funciona muy bien a altas temperaturas $\left(400^{\circ} \mathrm{C}\right)$ en el inicio de la reacción (1 h), con una conversión de casi el 100\% en alquenos. Por desgracia, el catalizador se desactiva rápidamente y al cabo de tres horas la actividad ha disminuido al 50\%. En cuanto a la selectividad, se observa que es consistente con respecto al material de partida, este decir, $11,7 \%$ de etileno, $1,3 \%$ de propileno, $6,68 \%$ de butenos y $86,84 \%$ de pentenos. Si comparamos los resultados de la deshidratación con el porcentaje de alcohol en el aceite de fusel (10,5\% de etanol, butanol 5,1\% y $84 \%$ de alcohol isoamílico), vemos que es consistente con lo que se obtuvo, excepto para los compuestos de la serie $\mathrm{C}_{4}$, que no corresponde al valor esperado, siendo un poco más importante, pero no significativo, lo cual está dentro de los rangos de error.

\subsection{Estudio de la deshidratación del aceite fusel con la zeolita HZSM-5 relación $\mathrm{Si} / \mathrm{Al}=\mathbf{2 8}$}

Una vez efectuado el estudio de la deshidratación de los alcoholes principales presentes en el aceite fusel por separado, se evalúa la deshidratación del aceite fusel como tal (figura 3). En primer lugar, se observó que al efectuar la reacción a una temperatura de $200^{\circ} \mathrm{C}$ se produce una transformación de los reactivos en un $90 \%$ y se obtienen las series $\mathrm{C}_{2}, \mathrm{C}_{3}, \mathrm{C}_{4} \mathrm{y} \mathrm{C}_{5}$. A medida que se 


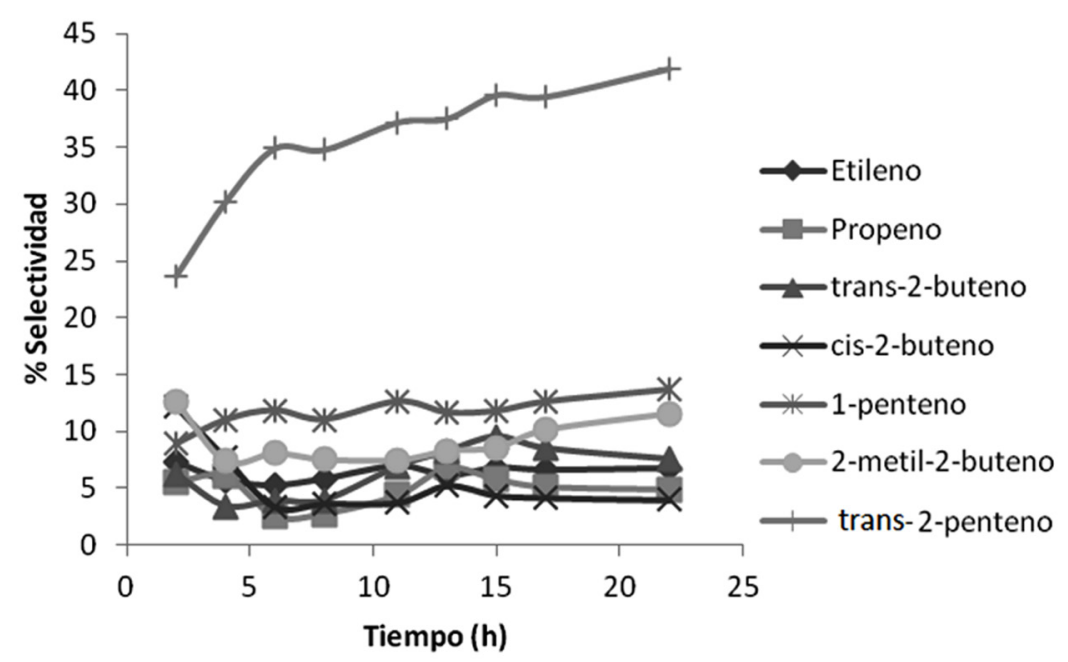

Figura 3. Evolución de la selectividad en función del tiempo para la deshidratación del aceite fusel. Masa del catalizador $0.4982 \mathrm{~g}$ con $3.0441 \mathrm{~g}$ de carborundo y un flujo de inyección del alcohol de $0.5 \mathrm{~mL} / \mathrm{h}$ y un flujo de gas de arrastre de $\mathrm{N}_{2}$ de $28 \mathrm{~mL} / \mathrm{min}$.

avanza en la reacción, se nota una desactivación de la zeolita, al constatar un aumento de alcoholes. Cuando se lleva a cabo la reacción a $260^{\circ} \mathrm{C}$, hay una transformación del $100 \%$, pero también se observa una desactivación lenta en función del tiempo, debido posiblemente a la formación de agua, producto de la deshidratación en el medio de reacción. De todas formas hay que decir que este catalizador sigue siendo activo por más de 24 horas. Con respecto a los productos encontrados en la reacción de deshidratación de aceite de fusel, se puede ver en la figura que el cis-2-penteno, el isómero más estable, es el producto mayoritario con una selectividad del orden de $40 \%$.

Ahora bien, teniendo en cuenta el papel que juegan los sitios ácidos y básicos planteados por varios autores Knozinger \& Scheglila (1970b), el mecanismo de coordinación en estas reacciones de deshidratación del aceite fusel, siguen un

(1)

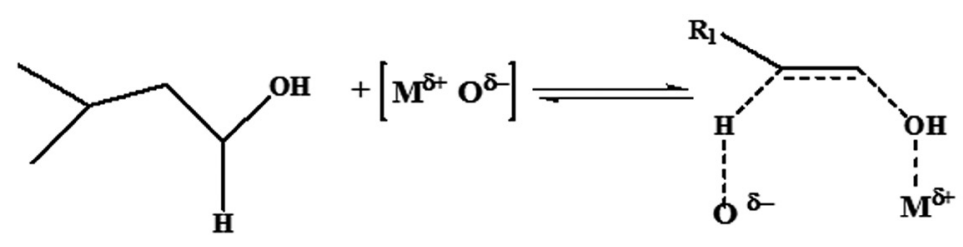

(2)

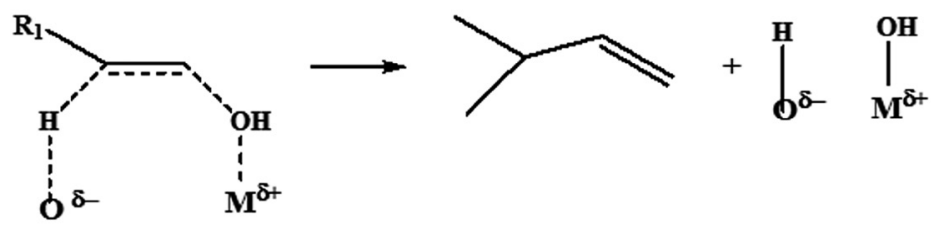

(3)

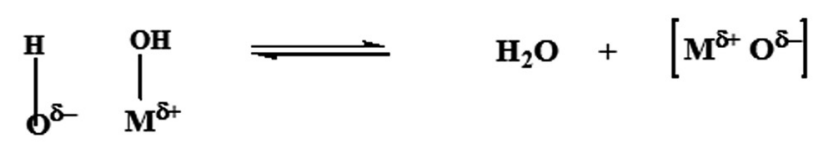

Figura 4. Mecanismo propuesto para la deshidratación del alcohol isoamílico sobre HZSM-5 zeolita. 
proceso de eliminación bimolecular o tipo $\mathrm{E}_{2}$, lo que implica un debilitamiento del enlace $\mathrm{CO}$ en el sitio ácido para lograr la eliminación concertada del $\mathrm{OH}$ y extracción del átomo de $\beta$-hidrógeno en un sitio básico. Estos resultados indican que cuando existe una posibilidad para la formación de diferentes isómeros geométricos, el más estable se forma predominantemente. Por lo tanto, la formación preferente de cis-2-penteno en esta reacción de deshidratación del alcohol isoamílico es consistente con las reacciones de deshidratación previamente reportadas para el butanol (tasa de conversión baja), en presencia de catalizadores con sitios ácidos de Lewis y de Brønsted, Knözinger et al. (1972a), Dabbagh \&Davis (1988), y Berteau et al. (1991), pudiéndose proponer un mecanismo tipo $\mathrm{E}_{2}$.

Este mecanismo concertado de reacción para la deshidratación de alcohol isoamílico que se muestra en la figura 4, incluye la adsorción no disociativa de alcohol sobre los sitios activos (paso 1), que constan de un par ácido-base conjugado que interactua de manera concertada con el grupo $\mathrm{OH}$ y un hidrógeno a-alcohol isoamílico (paso 2). Estos sitios activos están contituidos por un catión $(\mathrm{M} \mathrm{d}+)$ y un anión de oxígeno $(\mathrm{O} \mathrm{d}-)$, en el que el catión es $\mathrm{H} \mathrm{d}+$ para un sitio ácido de Brønsted. La adsorción del alcohol isoamilo es seguido por una eliminación concertada (E-2) para formar isómeros de penteno y dos grupos $\mathrm{OH}$ vecinales. Cuando $\mathrm{M}$ $\mathrm{d}+$ es un sitio ácido de Lewis, el $\mathrm{H}_{2} \mathrm{O}$ desorbida por recombinación de estos grupos hidroxilo (etapa 3), para volver a formar el enlace metal-oxígeno, que ha sido escindido en el paso 2 .

\section{Conclusiones}

La presencia de agua en mezclas que forman azeotropos es un factor limitante en la deshidratación de aceite de fusel y su separación es difícil e incrementa el costo, lo que hace que el proceso inviable. Desde este punto de vista, es indispensable utilizar estos productos en su estado crudo, sin ningún tratamiento previo. En este sentido, este trabajo se enfocó en el tratamiento del aceite fusel crudo, probando diferentes catalizadores. Cuando se utiliza un sólido ácido como la zeolita MFI, la cual posee una acidez intermedia, se hace necesario emplear temperaturas por encima de los $400^{\circ}$ C para observar una transformación significativa y aunque los rendimientos que se obtienen son buenos a alta temperatura (por encima de $450^{\circ}$ C) no es completa y se además se observa una desactivación en función del tiempo.

Por su parte, para la zeolita ZSM-5 con relación $\mathrm{SiO}_{2} / \mathrm{Al}_{2} \mathrm{O}_{3}$ de 1000 , la reacción se logra llevar a cabo pero a altas temperaturas. Lo anterior mostró que es esencial utilizar un sólido fuertemente ácido para alcanzar el efecto transformación de fusel. Efectivamente, cuando se trabaja con la zeolita ZSM-5 relación $\mathrm{SiO}_{2} / \mathrm{Al}_{2} \mathrm{O}_{3}$ de 28, un sólido fuertemente ácido, se obtienen los mejores resultados a temperaturas del orden de $\operatorname{los} 250^{\circ} \mathrm{C}$. En este caso, la composición de los productos obtenidos corresponde al producto más estable, es decir, a la formación principal de cis-2-penteno. Se observa igualmente la formación de otros productos isómeros de la familia como el 3-metil-1-buteno, 2-metil-1-buteno, 1-penteno, 2-metil-2-buteno y trans-2-penteno. Finalmente, hay que decir que se evidencia una ligera desactivación en el tiempo después de 6 horas de reacción.

\section{Agradecimientos}

Agradecimientos al laboratorio de investigación LACCO de la Université de Poitiers, Francia por permitir el análisis de los productos y a la Universidad del Valle por la financiación económica de esta estancia.

\section{Referencias bibliográficas}

Baertsch, C.D., Komala, K.T., Chua, Y-H., \& Iglesia, E. (2002). Genesis of Brønsted Acid Sites during Dehydration of 2-Butanol on Tungsten Oxide Catalysts. Journal of Catalysis $205,44-57$

Bandres, M., de Caro, P., Thiebaud-Roux, S., \& Borredon, M-E. (2011). Green syntheses of biobased solvents. Comptes Rendus Chimie C. R. Chimie 14 (7-8), 636-646 
Bakshi, K.R. \& Gavalas, G.R. (1975). Characterization of acid-base catalysts by calorimetric titration: I. Correlation with alcohol dehydration activity. Journal of Catalysis 38 (13), 312-325

Bryant, D.E. \& Kranich, W.L. (1967). Dehydration of Alcohols over Zeolite Catalysts. Journal of Catalysis 8, 8-13

Beers, A.E.W., Spruijt, R.A., Nijhuis, T.A., Kapteijn, F., \& Moulijn, J.A. (2001). Esterification in a structured catalytic reactor with counter-current water removal. Catalysis Today 66, 175-181.

Berteau, P., Delmon, B., Dallons, J-L., \& Van Gysel, A. (1991). Acid-base properties of silicaaluminas: use of 1-butanol dehydration as a test reaction. Applied Catalysis 70, 307-323

Bewa, H. \& Alfos, C. (2005). Le marché des agrosolvants. Oléagineux, Corps Gras, Lipides, 12 (4), 295-298

Cruz J.D. La caña de azúcar. http://www. fedebiocombustibles.com/v3/nota-web-id-1431. htm 27/11/2013

Dabbagh, H.A., \& Davis, B.H. (1988). Catalytic Conversion of Alcohols: The Impact of Inductive Effect for Secondary Alcohol Dehydration. Journal of Catalysis 110, 416-418

Dalpozzo, R., De Nino, A., Maiuolo, L., Procopio, A., Nardi, M., Bartoli, G., \& Romeo, R. (2003). Highly efficient and versatile acetylation of alcohols catalyzed by cerium (III) triflate. Tetrahedron Letters 44 (30), 5621-5624.

Dôrmô, N., Bélafi-Bakô, K., Bartha, L., Ehrenstein, U., \& Gubicza, L. (2004). Manufacture of an environmental-safe biolubricant from fusel oil by enzymatic esterification in solvent-free system. Biochemical Engineering Journal 21, 229-234

De Castro, H.F., Moriya, R.Y., Oliveira, P.C., \&Soares, C.M.F. (1999a). Fusel Oil as Precursor for Aroma Generation by Biotransformation Using Lipase Scientific Note. Applied Biochemistry Biotechnology 77-79, 817-826.
De Castro, H.F. \& Anderson, W.A. (1995b). Fine Chemicals by Biotransformation Using Lipases. Quimica Nova 18, 544-554.

Ewell, R.H. \& Hardy, P.E. (1941). Isomerization Equilibrium among the Branched Chain Pentenes. Journal of the American Chemical Society 63, 3460.

Frilette, V.J., Mower, E.B., \& Rubin, M.K. (1964). Kinetics of Dehydration of ter -Butyl Alcohol Catalyzed by Ion Exchange Resins. Journal of Catalysis 3, 25-31

Gates, B.C. (1992). Catalytic Chemistry. New York: John Wiley and Sons Inc., 371-373.

Gates, B.C., Wisnouskas, J.S., \& Heath Jr, H.W. (1972). The dehydration of t-butyl alcohol catalyzed by sulfonic acid resin. Journal of Catalysis 24 (2), 320-327

Ignace, J.W. \& Gates, B. C. (1973). Transport and Reaction of t-Butyl Alcohol in H-Mordenite Pores. Journal of Catalysis 29, 292-295

Güvenc, A., Kapucu, N., Kapucu, H., Aydoğan, Ö., \& Mehmetoğlu, Ü. (2007). Enzymatic esterification of isoamyl alcohol obtained from fusel oil: Optimization by response surface methodology. Enzyme and Microbial Technology $40,778-785$.

Haering, E.R. \& Syverson, A. (1974). Adsorption Studies at Reaction Conditions-Catalytic Dehydration of t-Butyl Alcohol. Journal of Catalysis 32, 396-414

Hoek, I., Nijhuis, T.A., Stankiewicz, A.I., \& Moulijn, J.A. (2004). Kinetics of solid acid catalyzed etherification of symmetrical primary alcohols: zeolithe BEA catalyzed etherification of 1-octanol. Applied Catalysis A 266, 109-116.

Ignace, J.W. \& Gates, B.C. (1973). Transport and Reaction of f-Butyl Alcohol in H-Mordenite Pores. Journal of Catalysis 29, 292-295 
Jermy, B.R., \& Pandurangan, A. (2005). A highly efficient catalyst for the esterification of acetic acid using n-butyl alcohol. Journal of Molecular Catalysis A 237, 146-154.

Knözinger, H., Buhl, H., \& Kochloefl, K. (1972a). The dehydration of alcohols on alumina: XIV. Reactivity and mechanism. Journal of Catalysis 24, 57-68.

Knözinger, H. \& Scheglila, A. (1970a). The Dehydration of Alcohols on Alumina XII. Kinetic isotope Effects in the Olefin Formation from Butanols. Journal of Catalysis 17, 252-263

Makgoba, N.P., Sakuneka, T.M., Koortzen, J.G., Van Schalkwyk, C., Botha, J.M., \& Nicolaides, C.P. (2006). Silication of g-alumina catalyst during the dehydration of linear primary alcohols. Applied Catalysis A: General 297, 145-150

Nijhuis, T.A., Beers, A.E.W., Kapteijn, F., \& Moulijn, J.A. (2002). Water removal by reactive stripping for a solid-acid catalyzed esterification in a monolithic reactor. Chemical Engineering Science 57, 1627-1632

Norris, J.F., \& Reuter, R. (1927). The rearrangement of isopropyl ethylene to trimethyl ethylene and the pyrogenic decomposition of pentene-2 and trimethyl ethylene. Journal of the American Chemical Society 49, 2624-40.

Osorio-Viana, W., Duque-Bernal, M., Fontalvo, J., Dobrosz-Gómez, I., Gómez-García, M.A. (2013). Kinetic study on the catalytic esterification of acetic acid with isoamyl alcohol over Amberlite IR-120. Chemical Engineering Science 101, 755-763

Özgülsün, A., \& Karaosmanoglu, F. (1999a). Environmentally Friendly Lubricating Oil Candidate. Applied Biochemistry Biotechnology 77, 83-89.

Özgülsün, A., Karaosmanoglu, F., \& Tüter, M. (2000b). Esterification Reaction of Oleic Acid With a Fusel Oil Fraction for Production of Lubricating Oil. Journal of the American Oil Chemists'Society 77, 105-110.
Patidar, P., Mahajani, S-M. (2012). Esterification of fusel oil using reactive distillation - Part I: Reaction kinetics. Chemical Engineering Journal 207-208, 377-387

Peters, T.A., Benes, N.E., Holmen, A., \& Keurentjes, J.T.F. (2006). Comparison of commercial solid acid catalysts for the esterification of acetic acid with butanol. Applied Catalysis A 297, 182-188.

Pizzio, L.R., \& Blanco, M.N. (2003). Isoamyl acetate production catalyzed by H3PW12O40 on their partially substituted Cs or K salts. Applied Catalysis A General 255 (2), 265.

Garcia E. (2013). Colombia sube producción y mezcla obligatoria de biocombustibles. http:// www.reporteenergia.com/noticias/index. php?option $=$ com_content $\&$ view $=$ article \&id $=4904$ : colombia-sube-produccion-y-mezclaobligatoria-de-biocombustibles\&catid=39: energi as-alternativas \&Itemid= 62 27/11/2013

Shi, B., Dabbagh, H.A., \& Davies, B.H. (1999). Alcohol dehydration. Isotope studies of the conversion of 3-pentanol. Journal of Molecular Catalysis A: General 141, 257-262.

Sun, J., Yu, B., Curran, P., Liu, S-Q. (2012). Lipase-catalyzed transesterification of coconut oil with fusel alcohols in a solvent-free system. Food Chemistry 134, 89-94

Tanabe, K., Misono, M., Ono, Y., \& Hattori, H. (1989). Catalytic activity and selectivity. In: Stud. Surf. Sci. Catal., 5, (Chapter 4)

Teo, H., \& Saha, B. (2004). Heterogeneous catalyzed esterification of acetic acid with isoamyl alcohol: kinetic studies. Journal of Catalysis 228 (1), 174-182.

Vulfson, E.N. (1993). Enzymatic synthesis of food ingredients in low-water media. Trends Food Science Technology 4, 209-215.

Watanabe, Y., Shimada, Y., Sugihara, A., Noda, H., Fukuda, H., \& Tominaga, Y. (2000). Continuous production of biodiesel fuel from vegetable oil using 
immobilized Candida Antarctica lipase. Journal of the American Oil Chemists' Society 77, 355-360

Welsh, F.H., Murray, W.D., Williams, R.E., \&Katz, I. (1989). Microbiological and enzymatic production of flavor and fragrance chemicals. Critical Reviews in Biotechnology 9 (2), 105-169.
Welsh, F.W. \& Williams, R.E. (1989). Lipase Mediated Production of Flavor and Fragrance Esters from Fusel Oil. Journal Food Science 54, $1565-1568$. 